\title{
TECNICA DI PRELEVAMENTO E PURIFICAZIONE DEI GAS NATURALI E VULCANICI
}

\author{
M. Santangelo
}

Recentemente sono stati pubblicati $(1,2)$, i risultati di una ricerca sulla composizione isotopica dell'Argon contemto nei gas provenienti dallinterno della crosta terrestre.

In lavori di questo genere uno dei problemi fondamentali è costituito dalla operazione di captazione sul terreno dei campioni e la diffcoltà più grossa sta nel fatto che, specie nel caso dei vulcani, è molto problematico evitare l'inquinamento da parte dell'aria atmosferica, e ció sia per la inadegruatezza dei dispositivi di raccolta, sia anche, e questo è più grave, perché l'inquinamento può verificarsi per circolazione sotterranea di aria prima ancora che $i$ gas vengano in superficie (3).

Agriungasi che questi gas in genere si presentano con elevata temperatura, 200-300 "C, la qui la necessità che l'apparecchiatura ahbia particolari requisiti.

Le difficolti di cui sopra, naturalmente, scompaiono quando il gas viene incondottato a profonditì dell'orline di qualche centinaio di metri, cosi come arviene per i soffioni horaciferi di Larderello (Toscana). Per le ricerche di cui si è fatto cenno (l. e.), presso l'Istituto Nazionale di Geofisica in collahorazione con il Centro di Fisica Nucleare di Roma, i stata studiata e realizzata una attrezzatura per il prelevamento e la purificazione dei gas naturali allo scopo di preparare dei campioni di Argon da analizzare con lo spettrografo di massa +4 .

Gas vulcanici. - La tecnica impiegata rariava a seconda che si tratlasse di emanazioni da crepacci di vulcani attivi o di fumarole subacquee: nel primo caso fu costruito un sistema a bombola con apertura manovrabile a distanza. La bombola del rolume di circa 10 litri (vedi figr. 1) è munita di un dispositivo di chinsura di cui alla fig. 2. Questo dispositivo normalmente resta chiuso a tenuta di vuoto. La hombola protetta da un cappuccio metallico viene calata a mezzo di un 
cavo di acciaio alla profondità massima raggiungibile nel crepaccio da cui provengono $i$ gas che si vogliono captare.

Lina volta introdotta la bombola, da una distanza clic nei nostri prelevamenti si aggrirava attorno agli $8-10$ metri, veniva aperta a mezzo di un cavo con anima di acciaio la valvola azionata clalla leva $A$ di fig. 2: dopo qualche minuto la hombola si riempiva dei gas circolanti nel crepaccio: il vuotometro indicava l'avvenuto ricmpimento.

Per questa operazione si è cercato di sceglicre quelle spaccature che presentavano le caratteristiche più opportune perchè $i$ gas captati fossero quanto più possibile esenti da inquinamento di aria.

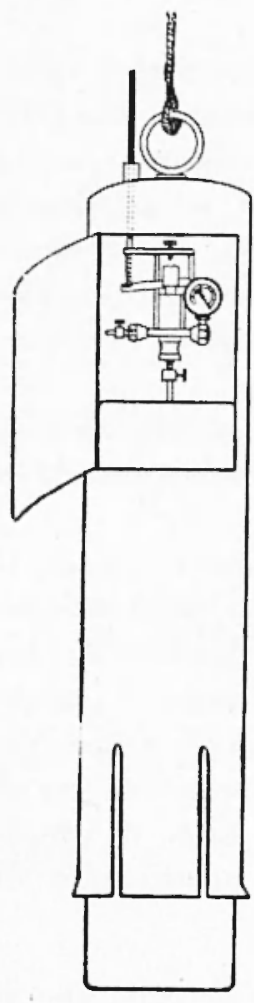

Fig. 1

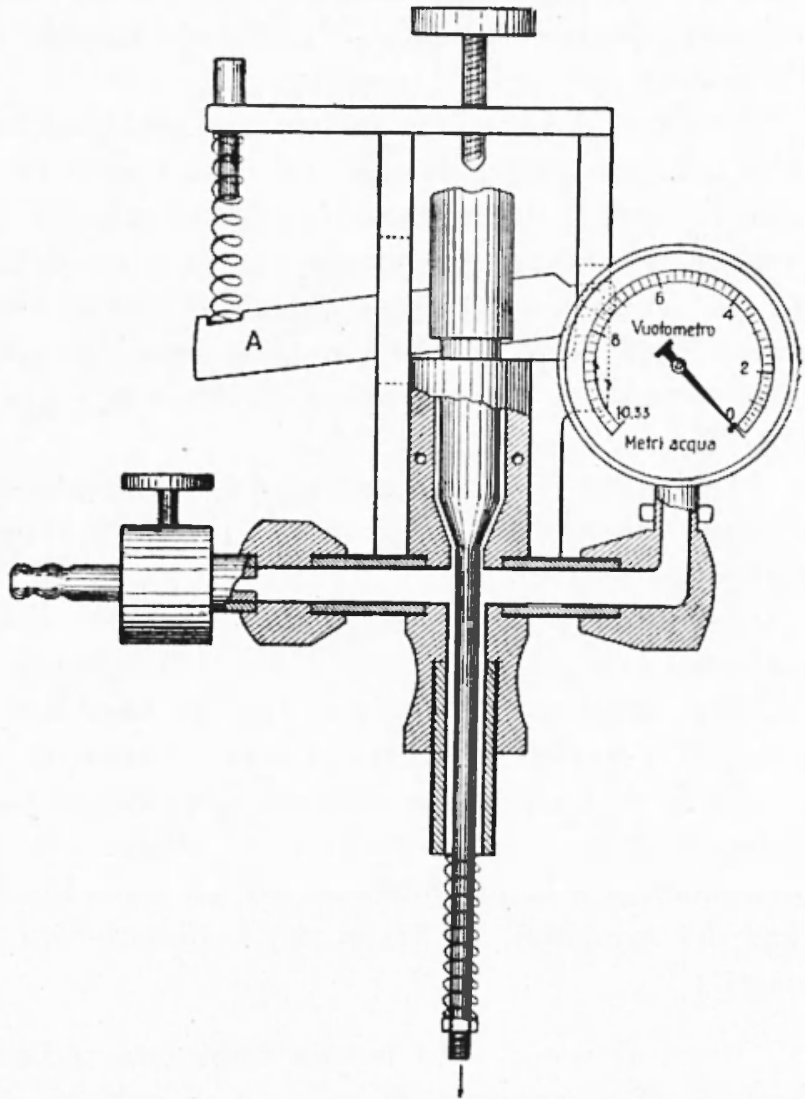

Fig. 2

Con questa tecnica furono fatti prelevamenti sull'Etna; ne fu tentato anche uno allo Stromboli, ma l'operazione non si poté effettuare con le modalità sopra descritte per il lancio intermittente da parte del vulcano di lapilli infuocati, masse incandescenti e gas tossici. 
Fumarole subacquee, - Il prelevamento si presenta più agevole e con risultato alquanto più sicuro nel caso di fumarole subacquec. I gas gorgoglianti nell'acqua sono raccolti in una campana di circa 20 litri (fig. 3) che viene c:alata a mezzo di una picrola uru, girevole e facilmente trasportabile, nollo specchio di acqua fino ai $2 / 3$ della sua altezzil. Del ghiaccio posto in un cilindro innestato sulla campana, provoca la condensizione del valpore d'acqua trascinato dai was nel tubo di rame a spirale immerso in esso: in questo modo l'acqua di condensazione ricade, mentre $\mathrm{i}$ gas residui pervengono attraverso un tuho di romma al un sistema (fig. 4 ) costituito da 2 bocce contenenti una soluzione di $\mathrm{NaOH}$ a circa $30 \%$, preventivamente disacrata a caldo $\mathrm{e}$ sotto vuoto. Il manometro $B$ serve ai: inclicare la pressione del gas rispetto a quella itmosferica.

L'operazione procedeva nel seguente modo: si faceva riempire la prima hoccia, quindi si scuoteva la soluzione per agevolare la reazione:

$2 \mathrm{NaOH}+\mathrm{CO}_{2}-\mathrm{Na}_{\mathrm{aCO}} \mathrm{CO}_{3}+\mathrm{H}_{2} \mathrm{O}$, cosi alla fine era possibile assorbire nella quasi totalita la $\mathrm{CO}$, che in questi gas puo raggiungere

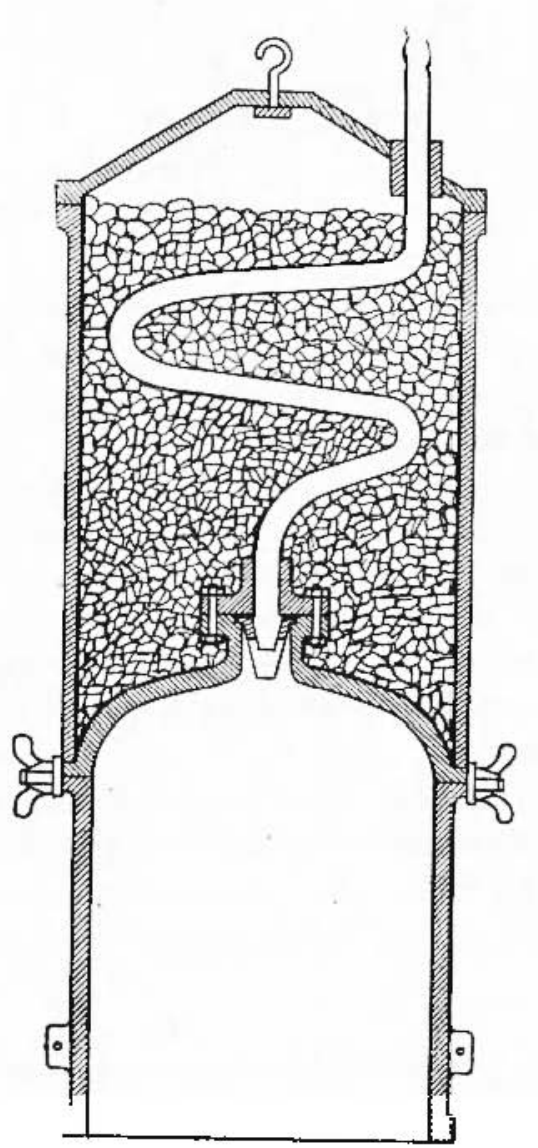

Fig. 3 il $95-98 \%\left({ }^{3}\right)$ e raccogliere il gas residuo senza che ci fosse pericolo di contaminazione da parte dell'aria atmosferica.

Campioni sono stati prelevati ad Agnano Terme, Solfatara di Pozzuoli e Bullicame di Viterbo.

Preparazione del campione di Argon. - I gas vulcanici e fumarolici contengono prevalentemente $\mathrm{H}_{2} \mathrm{O}, \mathrm{CO}, \mathrm{N}$, e talvolta $\mathrm{H}_{n} \mathrm{~S}, \mathrm{CH}_{4}$, 


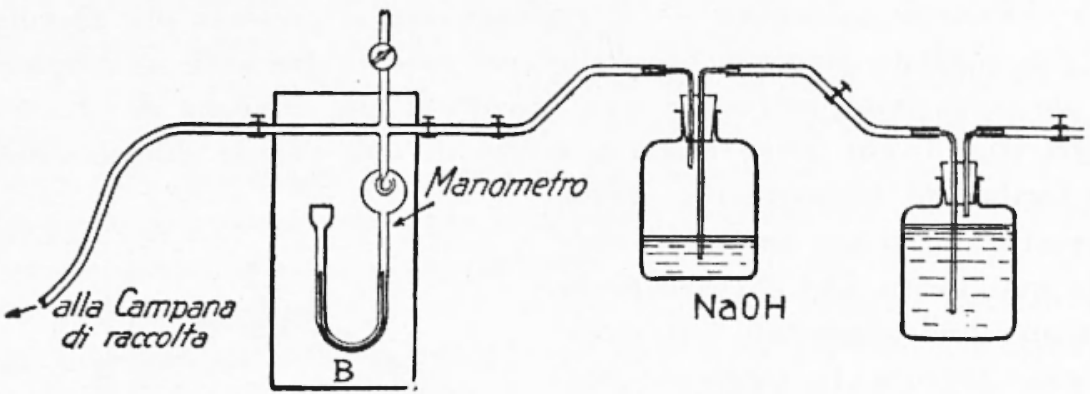

Fig. 4

$H_{2}$ e gas rari. 11 metorlo usato per la purificazione è quello classico $(\bullet)$ : per ottenere i campioni di Argon si sfrutta la propricti dei gas nobili di non reagire chimicamente: il problema si riduce ad una successiva eliminazione di tutti gli altri gas presenti. Non è necessario che questa eliminazione sia quantitativa poiché le misure dei rapporti tra ghi isotopi dell'Argon con lo spettrografo di massa non vengono disturbate da nessuna delle masse riportate.

L'H.O residua viene eliminata facendo passare il gas attraverso tubi ad U contenenti $\mathrm{CaCl}_{\circ}$ e $P_{2} \mathrm{O}_{5}$, la $\mathrm{CO} .2$ mediante calce sodata, e l'ossigeno, se presente, si fa assorbire in un forno di Pirex, da $C u$ metallico a $350^{\circ} \mathrm{C}$.

L'azoto viene trattenuto sotto forma di azoturo in un forno metallico dove è contenuto $C a$ metallico alla temperatura di circa $700{ }^{\circ} \mathrm{C}$ (fig. 5). Il gas residuo composto prevalentemente da Argon e dagli altri gas rari, viene aspirato dal forno a $C a$, dove alla fine si raccorlic,

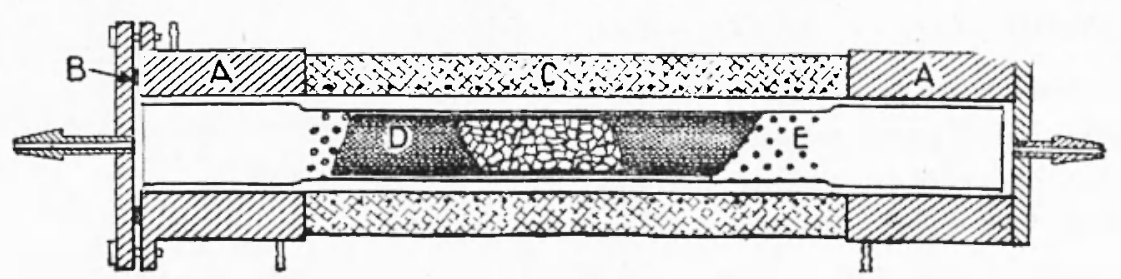

Fig. 5

Forno a $\mathrm{Ca}$

$A$ - Manicotti di raffreddamento a circolazione di acqua

$B$ - Guarnizione ad anello di nomma per le tenuta del ruoto

$C$ - Riscaldatore elettrico

D - Cilindro di reticella di ferro contenente le tornitura di Ca

$E$ - Tubo di acciaio esterno a $D$. con fori per il passaggio del gas 
in un recipiente in cui si fa adsorbire da carbone attivo raffreddato con aria liquida: da qui si manda in un palloncino di vetro di $100 \mathrm{~cm}^{3}$.

In fig. 6 ì schematizzato l'insieme del dispositivo per la purifcazione dei gas.

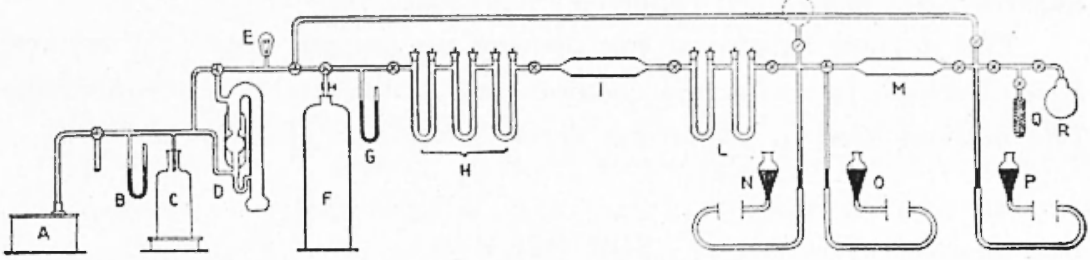

Fig. 6

$A$ - Pompa rotativa - vuoto preparatorio

$B$ e $G-$ Manometri ad $\mathrm{Hg}$

$C$ - Serbatoio per il vuoto

$D$ - Pompa in pyrex a diffusione di $H g$

$E$ - Vacuometro di Pirani

$F$ - Bombola contenente il gas da purificare

$\mathrm{H}$ - Tubi ad $\mathrm{L}$ contenenti $\mathrm{CaCl_{2 }}$ e calce sodata

$I$ - Forno in pyrex a rame

$L$ - Tubi ad U con $P . O$,

$N, O . P$ - Pompe di Toepler

$M$ - Forno metallico a $C a$

$Q$ - Recipiente con $C$ attivo

$R$ - Palloncino in cui si raccoglie l'Argon e $\mathrm{i}$ gas rari

Mi è gradito ringraziare i colleghi dottori G. Boato e G. Careri per l'aiuto prestato durante la preparazione delle apparecchiature qui descritte.

Roma - Istituto Nazionale di Geofisica - Gennaio 1952.

\section{RIASSUNTO}

In lavori precedenti sono stati resi noti i risultati di analisi sulla composizione isotopica dell'Argon contenuto nei gas naturali.

Nella presente nota vengono brevemente esposti $i$ criteri tecnici seguiti nella progettazione ed esecuzione delle apparcchiature necessarie al prelevamento dei gas stessi. Si descrivono le tecniche impiegate per la captazione sia dei gas vulcanici che di quelli delle fumarole subacquee. Fiene infine succintamente esposto il procedimento per la preparazione dei campioni di Argon e dato uno schema del dispositivo usato per tale operazione. 


\section{$S U M M A R Y$}

The results of mass-spectrometric analysis of the Argon content in natural gases have been reported in previous papers.

The devices employed for piching up natural gases are reported here. Methods for collecting volcanic and fumarolic gases are described. The method used in preparing Argon samples is also summarized.

\section{BIBIIOGRAFIA}

(1) G. Bonto-G. Careri-G. Nexcini-M. Santanglio, Aunali di Geofisica. IV.1-1951.

(2) G. Bonto - G. Canfri - M. Santangelo, Nuovo Cimento. IX-1-1951.

(3) Seonc1, Annali di Chimica Applicata. Vol. 32 fasc. 12, 1912 ; Day, Snejrero, Bullet. Geol. Soc. Volc. Am. 21, 573, 1913.

(t) G. Careri - G. Nencini, Nuovo Cimento. 7, 64, 1950.

(5) P. Pascal, Traite de chimie minerale. Paris, 1934. 